\title{
Axial Flow Fan Tip Leakage Flow Control Using Tip Platform Extensions
}

\author{
Ali Aktürk* and Cengiz Camci ${ }^{\dagger}$ \\ Turbomachinery Aero-Heat Transfer Laboratory \\ Department Aerospace Engineering \\ Pennsylvania State University \\ University Park, PA 16802
}

\begin{abstract}
Performance of an axial flow fan unit is closely related to its tip leakage mass flow rate and the level of tip/casing interactions. The present experimental study uses a Stereoscopic Particle Image Velocimeter to quantify the three dimensional mean flow observed near the blade tip at just downstream of a ducted fan unit. After a comprehensive description of the exit flow from the baseline fan, a number of novel tip treatments based on custom designed pressure side extensions are introduced. Various tip leakage mitigation schemes are introduced by varying the chordwise location and the width of the extension in the circumferential direction. The current study shows that a proper selection of the pressure side bump location and width are the two critical parameters influencing the success of each tip leakage mitigation approach. Significant gains in axial mean velocity component are observed when a proper pressure side tip extension is used. It is also observed that a proper tip leakage mitigation scheme significantly reduces the tangential velocity component near the tip of the axial fan blade. Reduced
\end{abstract}

\footnotetext{
*Graduate Research Assistant, e-mail: aua162@psu.edu

$\dagger$ Professor of Aerospace Engineering, corresponding author, e-mail: cxc11@psu.edu
} 
tip clearance related flow interactions are essential in improving the energy efficiency of ducted fan systems. A reduction or elimination of the momentum deficit in tip vortices are also essential to reduce the adverse performance effects originating from the unsteady and highly turbulent tip leakage flows rotating against a stationary casing.

\section{Nomenclature}

$\beta_{1} \quad$ Blade section inlet angle

$\beta_{2} \quad$ Blade section exit angle

c Chord length

c Chord length

$C C D \quad$ Charge-Coupled Device

$\epsilon \quad$ Experimental uncertainty

$h \quad$ Blade height

$N \quad$ Number of SPIV speckle images

PLIF Phosphorescence Laser Ind. Flourosence

$p \quad$ Static pressure

$r \quad$ Radial position ( $\mathrm{r}=0.0$ at axis of rotation)

$R_{H u b} \quad$ Hub radius

$R_{\text {Tip }} \quad$ Tip radius

SPIV Stereoscopic Particle Image Velocimeter

$\sigma \quad$ Standard Deviation

$t \quad$ Effective tip clearance in inches 


$\begin{array}{ll}U_{a} & \text { Averaged axial velocity } \\ x & \text { Radial direction, see Figure } 4 \\ y & \text { Axial direction, see Figure } 4 \\ z & \text { Tangential direction, see Figure } 2\end{array}$

\section{Introduction}

The flow field between the stationary casing and rotor tip of an axial fan is complex because of the interaction of the leakage flow, annulus wall boundary layer and rotor wake. Inherent pressure difference between the pressure side and suction side of blade tip generates a tip leakage flow that is responsible for a substantial portion of aerodynamic losses in axial flow fans. The leakage flow also rolls into a highly three dimensional tip leakage vortex with significantly turbulent and unsteady flow features in each passage. Tip leakage vortex is a complicated flow phenomenon that is one of the dominant mechanisms of noise generation by unsteady interactions in a turbomachinery system. It is also one of the major energy loss mechanisms for axial flow fan systems. Despite the close relation between the tip leakage flow and performance of axial fans, there has been limited amount of information about three dimensional flow structure of leakage vortex in open literature [1-4].

Inoue and Kuroumaru et al. [5] made detailed flow measurements before and behind an axial flow rotor with different tip clearances. In their study, they investigated the clearance effect on the behavior of tip leakage flow. Furukawa and Inoue et al. [6] also investigated breakdown of tip leakage vortex in a low speed axial flow compressor. Reducing tip leakage mass flow rate improves the aerodynamic performance of axial flow fans and compressors. 
Implementation of treatments in the non-rotating part over the blade tip is also an efficient way of tip leakage flow reduction. References [7] and [8] investigate different casing treatments for axial flow compressors.

The wake developed from an axial flow fan has a strong influence on the system performance. It is a significant source of aerodynamic loss and affects the efficiency and vibration characteristics. References [9-11] deal with extensive investigations of the wake flow features such as mean velocities, turbulence and decay characteristics on turbomachinery performance.

Stereoscopic Particle Image Velocimetry (SPIV) is an effective way of measuring all three velocity components of an instantaneous flow field over a selected area [12-16]. A comprehensive discussion of the specific stereoscopic PIV technique used in this paper is given by Kahveci and Camci in [13-15]. Yoon and Lee et al. [16] investigated the flow structure around an axial flow fan using the SPIV technique. The time averaged results clearly show the evolution and dissipation of tip vortices. Yen and Lin et al. [17] analyzed exit flow performance and properties of an axial flow fan with winglet-blades at various impeller angles using SPIV. The velocity profiles show the most stable and the best fan performance, resulting from winglet-blades which increase the lift and reduce the drag. Wernet et al. [18] made phase-locked three-dimensional Digital Particle Image Velocimetry (DPIV) measurements near the tip region of a low speed compressor rotor to characterize behavior of rotor tip clearance flow. Comparison of the DPIV measurements to Navier-Stokes flow simulations was also done.

Corsini et al. [19-22] presented the results of a computational study of an axial flow fan using "improved tip concepts". The first two endplates were with constant and variable 
thickness distributions while the last two were designed by combining the end-plates with a stepped gap on the tip. The investigation was based on a finite element Navier-Stokes solver for the physical interpretation of the detailed 3D leakage flow field.The specific fan performance experiments have shown that the improved tip concepts introduced a small performance de-rating, but the efficiency curves give evidence of an improvement with better peak performance and a wider high efficiency curve towards the rotor stall margin. An aero-acoustic investigation showed a reduction of the rotor aero-acoustic signature.

The computationally predicted loss coefficients in Corsini et al. [22] showed that the highest loss regions were always observed near the leakage vortex core. The computational comparison of mechanical energy loss within the gap showed that the tip end-plates lead to a reduction of mechanical energy loss within the gap.

The Present experimental study used stereoscopic particle image velocimetry measurements (SPIV) at the exit of the seven bladed axial fan rotor.Fan rotor used for the experiment is widely used in air conditioning industry. All of the experiments were performed at constant rotational speed of the fan. $(859 \mathrm{rpm})$.

The main objectives of the current study are summarized as follows:

- To investigate the characteristics of tip leakage flow and tip vortex in an axial flow fan using a high spatial resolution and non-intrusive measurement technique. All three components of the tip region exit flow field are measured using SPIV technique.

- To eliminate the adverse effects of tip vortex by designing novel rotor tip geometries using pressure side extensions.

- To reduce the local leakage mass flow rate and momentum deficit in tip vortex driven 
flow structures.

- To Increase the energy efficiency of axial-flow fan systems.

- To provide high quality phase-locked instantaneous flow data for further studies in aero-acoustics of tip treated axial flow fans.

\section{Experimental Set-up}

Test rig: The test rig consisting of an axial flow fan, a mock-up unit and an electric drive system as shown in figure 1 was designed to investigate the rotor exit flow phenomena using a stereoscopic PIV system. The set-up has also provisions for seeding the fan flow field with a smoke generator using a fluidized bed. The smoke generator is located near the inlet section of the mock-up unit where a perforated plate controlling the fan loading is mounted. The electric motor driving the fan rotor is speed controlled by an AC inverter unit. The current phase-locked SPIV measurements are triggered by using an optical onceper-revolution device located near the hub of the rotor inlet. An infrared beam is reflected from a highly reflective surface attached to the rotor hub. This once-per-rev pulse provides a phase-locked triggering of the SPIV data collection system. The relative position of the rotor can be adjusted accurately in relative to the position of the laser light sheet that contains the rectangular SPIV measurement area.

Ducted Fan: Figure 2 shows seven bladed axial flow fan unit including the orientation of the SPIV system components. The geometric specifications are presented in figure 3. A perforated plate at the inlet section of the mock-up unit is used for creating realistic fan 
loading conditions. The tips of the blades are modified through a removable "operation window" as shown in figure 1 . Only one blade tip out of seven blades is modified since the current SPIV system is capable of measuring in the immediate vicinity of a "selected" blade tip due to the phase-locked and instantaneous nature of the SPIV measurements. A precision machined 0.030 inch $(0.762 \mathrm{~mm})$ thick thermoplastic layer was used for the selected tip platform design. The tip platform design was attached to the precision machined tip area in a non-intrusive way through the operation window as shown in figure 1 . The modifications made on the selected tip did not cause a measurable rotor balance problem since the rotor was manufactured out of relatively light weight thermoplastic material. The current rotor blades have serrated trailing edges for effective mixing of the blade boundary layers in the wake of each blade. The serrated trailing edges also provide an effective mixing of individual tip vortices with the wakes of the seven blades in the rotating frame of reference. It should be noted that the comparison of the flow with serrated trailing edges with smooth trailing edge flow is not the subject of the current paper.

Fan Performance: The performance of the fan unit is measured under three different loading conditions. The first performance point $\left(80 \mathrm{~m}^{3}, 140 \mathrm{~Pa}\right)$ shown in figure 4 is obtained by using a perforated steel plate having an open area ratio of 19.6\%. A second perforated plate using a slightly larger open area ratio of $42.5 \%$ provides the middle point $\left(280 \mathrm{~m}^{3} / \mathrm{min}\right.$, $88 \mathrm{~Pa})$ in the performance curve as shown in figure 4 . The third point with the highest volumetric flow rate at $340 \mathrm{~m}^{3} / \mathrm{min}$ is obtained when there is no perforated plate installed at the inlet section of the mock-up unit. The pressure change across the fan is measured by using pitot static holes mounted on all four sides of the mock-up unit. Wall-static pressures from all four sides are averaged. For the mass flow rate measurements, a hot-wire based 
volumetric flow measurement device is used at rotor downstream.

Stereoscopic PIV system: The PIV technique measures instantaneous velocity components of a flow field over a determined area. Small particles that go with the flow are introduced into the fluid flow, and the region of interest is illuminated by the light sheet provided by to subsequent Nd-Yag laser pulses lasting as short as a few nanoseconds. The subsequent step is the recording of the displacement of particles via two high sensitivity CCD cameras.

The scattered light from the seeding particles are recorded by two different cameras for simultaneous recording on digital media. The initial position of a particle is recorded on the first frame of the camera right after first laser pulse fires. In general, a typical duration of a laser pulse is about 30 nano seconds in a flow field similar to the current study. Its final position is recorded in the same way on the second frame of the same camera when the second laser pulse fires. The time interval between the two frames is usually determined by the mean flow speed in the area of investigation. The order of magnitude of this adjustable time separation between the two frames is "microseconds". Since the displacement of the particle and the time interval between the two subsequent laser pulses are known, the velocity of the particle can be calculated by the simple equation: speed=distance/time. A comprehensive explanation of this technique is given in references [12-16].

In 3D PIV, there is an additional camera viewing the field from a different angle. The two-dimensional image obtained by each camera is slightly different from each other, and they are afterwards combined to produce the three-dimensional velocity information. Stereoscopic vision principles are instrumental in this process of combining the two planar images obtained from the two cameras viewing the same flow-field simultaneously. The data re- 
duction in a stereoscopic PIV system requires the processing of four independent images from the two cameras. For 3D analysis, the $2 \mathrm{D}$ calibration images need additionally to be converted into 3D data by a Direct Linear Transform (DLT) model, in order to calculate the third component of the velocity. Correlation techniques are used to obtain raw vector maps out of image pairs taken during the experiments, and a number of calculation methods are used to evaluate these vector maps. In summary, the three dimensional space defined by the planar measurement area and the finite thickness of the laser sheet is analytically described in relation to the highly distorted images captured by the two CCD chips in each of the two cameras. The distortions observed on the planar CCD images are generated by the angled position of two cameras. For example, a perfect cube in the measurement space is seen as a distorted cube in each one of two images generated by two cameras.

The current study uses high sensitivity cameras that are essential in high speed flow measurements. Two of the 80C60 HiSense PIV/PLIF cameras with 1024 x 1280 pixels are used with 80 N57 personality module fitted to processor, and a Nikon micro-Nikkor 60/2.8 objective, for each camera. The calibration plate, the cameras, Nd-Yag pulsed laser unit and axial fan rotor blades are shown in figure 2 . Initial calibration procedure requires that the laser light sheet generated by the Nd-Yag laser is aligned with the calibration plate carrying a standard high precision square grid. 


\section{Experimental Methodology}

Measurement Domain: The axial (y), radial (x) and tangential (z) components of velocity profiles were simultaneously measured near the tip region of the fan under the influence of a few novel tip platform extensions designed throughout this investigation. Figure 5 shows the measurement domain which is a rectangle in the $\mathrm{x}-\mathrm{y}$ plane located at just downstream of the rotor. The $\mathrm{x}-\mathrm{y}$ plane is horizontal and contains the axis of rotation. Most of the SPIV distributions covered a span-wise region from $\mathrm{r} / R_{\text {Tip }}=0.6$ to $\mathrm{r} / R_{\text {Tip }}=1.3$. This measurement area corresponds to a region covering the last three quarters of the blade height including the tip region flow. The span-wise velocity distributions shown in Figures 8, 9, 10 and 11 are obtained at an axial position $46 \mathrm{~mm}$ away from the rotor exit plane. The radial direction is also marked with $\mathrm{r} / R_{\text {Tip }}$ in order to mark the exact position of the blade root and tip in the span-wise distributions of velocity. " $r / R_{\text {Tip }}$ " is approximately 0.33 at blade root location (hub).

Specific Rotor Positions for Phase-locked Measurements: The results from the custom designed tip platform extensions are compared to the results obtained from a baseline tip at two different tip clearance levels. All three components of the velocity vector were measured for 7 circumferential positions of the rotor (with respect to the SPIV measurement plane). These locations were chosen by dividing the rotor blade pitch into 7 equi-angular regions. Although the measurements were performed at seven positions, only the most pertinent data from three selected positions are presented. The selected positions include blade tip leading edge, mid-chord and trailing edge (locations 3,4,5). 
Statistical Stability of SPIV measurements: An adequate number (N) of SPIV speckle images should be recorded and processed for satisfying statistical requirements for accurate mean velocity measurements. The ensemble average of measured velocity components approaches to a true mean as $\mathrm{N}$ goes to infinity. During an ensemble averaging of finite number of instantaneous velocity measurements, it should be noted that their average may have a deviation from the true mean value. This deviation is closely related to the sample size $(\mathrm{N})$ which is a measure of the statistical stability of this ensemble averaging process. Table 1 shows deviations calculated from measured data for radial, axial and tangential velocity components obtained by averaging 50,100,150 and 250 PIV speckle images. Standard errors are calculated by using the central limit theorem [23]. The central limit theorem states that uncertainty can be approximately equal to the standard deviation of measured values $(\sigma)$ divided by $\sqrt{N}$ (i.e $\epsilon=\frac{\sigma}{\sqrt{N}}$ ). The uncertainties are estimated at locations of the highest

\begin{tabular}{cccc}
\hline \hline & Radial Velocity & Axial Velocity & Tangential Velocity \\
\hline $\mathrm{N}=50$ & $1.19 \times 10^{-1} U_{a}$ & $1.11 \times 10^{-1} U_{a}$ & $1.98 \times 10^{-1} U_{a}$ \\
$\mathrm{~N}=100$ & $7.47 \times 10^{-2} U_{a}$ & $6.44 \times 10^{-2} U_{a}$ & $1.28 \times 10^{-1} U_{a}$ \\
$\mathrm{~N}=150$ & $6.01 \times 10^{-2} U_{a}$ & $4.85 \times 10^{-2} U_{a}$ & $8.89 \times 10^{-2} U_{a}$ \\
$\mathrm{~N}=200$ & $4.77 \times 10^{-2} U_{a}$ & $3.97 \times 10^{-2} U_{a}$ & $7.93 \times 10^{-2} U_{a}$ \\
$\mathrm{~N}=250$ & $3.99 \times 10^{-2} U_{a}$ & $3.39 \times 10^{-2} U_{a}$ & $6.68 \times 10^{-2} U_{a}$ \\
\hline
\end{tabular}

Table 1: Uncertainties of ensemble averaged velocities components

standard deviations of individual components for each sampling image size. They are normalized by using averaged axial velocity $\left(U_{a}\right)$ calculated from the mass flow rate of the fan for the condition that experiments are conducted $\left(340 \mathrm{~m}_{3} / \mathrm{min}, 32 \mathrm{~Pa}\right)$. Note that the most Aktürk \& Camc1 FE-09-1205 
uncertainties are smaller than $9 \%$ for the ensemble image size more than 150 images.

Based on the examination of data convergence with the number of speckle images, sampling image size $(\mathrm{N})$ is selected to be 170. Figure 6 presents the influence of ensemble averaging sample size on the span-wise distribution of the most significant velocity component "axial component". The baseline blade tip is used in this experiment with a nominal tip clearance of $1 \%$ of the blade height. The span-wise region where $0.9 \leq r / R_{\text {Tip }} \leq 1.02$ is not significantly influenced from the choice of the ensemble averaging sample size. The sample sizes of 150, 170, 200 and 250 produce very similar span-wise distributions in this region near the tip. All SPIV experiments presented in figures 8 - 13 are conducted using an ensemble averaging sample size of 170 .

Tip Clearance Values: An effective control of tip leakage flow was achieved using different tip platform extensions. The term "control of tip leakage flow" in this study could be defined as the "minimization of tip leakage flow mass flow rate". Baseline profile was evaluated at two different tip clearance values of 0.1 inch and 0.135 inch corresponding to $1 \%$ and $1.35 \%$ of blade height. All of the "tip platform extensions" used in this study were evaluated at tip clearance values of 0.1 inch corresponding to $1 \%$ of the blade height.

The General Impact of Tip Platform Extensions: The main goal of this study is to minimize the tip leakage flow by interfering with the flow near the pressure side corner of the blade tip region. A new static pressure distribution near the blade tip section is established by the novel tip platform extension designs shown in figure 7 .

The impact of the suggested tip platform extensions is visible in the "magnitude enhancements" of the axial velocity component downstream of the tip region. It is also highly visible that the strong tangential velocity components induced by the baseline tip are almost 
eliminated by effective tip platform design. This observation is very clear especially near the tip diameter of the rotor exit flow

Geometrical Definition of the Five Tip Platform Extensions: The design idea behind the present tip platform extensions is based on estimating the blade chordwise position where the tip leakage potential is the highest for a given tip profile. It is obvious that the leakage potential is represented by the static pressure differential imposed by the shape design of the blade profile. Initial CFD based flow prediction is may be effective in finding the chordwise region where the leakage mass flow rate is maximum.

The tip platform extensions were designed by adding constant-thickness "pressure side bumps" of different designs to the base profile as shown in figure 7 . The thickness of the platform extended on the pressure side of the blade is about 0.030 inch $(0.762 \mathrm{~mm})$ for all designs used in this investigation. Initially, a wide bump between the trailing edge and the leading edge was suggested as Profile 1 in figure 7 . The maximum width of the bump was chosen as the same as the thickness of the airfoil at the bump centerline location. Three more tip platform extensions were derived from Profile 1 by dividing the bump width of Profile 1 into three almost equal chord-wise sections. The three new tip platform extensions are termed Profile 2 (near the trailing edge), Profile 3 (middle of the wide bump) and Profile 4 (almost mid-chord). The bump maximum widths were kept the same. Profile 5 is a derivative of the trailing edge bump named as Profile 2. Profile 5 uses a relatively small platform area when compared to all other tip platform extensions. The external contour of Profile 5 is almost parallel to the baseline profile except the end points where blending is suggested.

Only one rotor blade at a time out of seven blades was retrofitted with a suggested de- 
sign. Each experiment had six baseline blades and a seventh blade with the suggested tip platform extension. The effective clearance was kept same for all seven blades.

\section{Experimental Results and Discussion}

Figures $8,9,10$ and 11 show the results of the exit flow measurements of the axial flow fan at the highest volumetric flow rate condition defined as $\left(340 \mathrm{~m}^{3} / \mathrm{min}, 32 \mathrm{~Pa}\right)$. Figure 12 complements these results from a data set obtained at the minimum mass flow rate condition where the pressure loading is maximum $\left(80 \mathrm{~m}^{3} / \mathrm{min}, 140 \mathrm{~Pa}\right)$. All the velocity profiles are plotted at 1.811 inch $(46 \mathrm{~mm})$ downstream of the fan.

In addition to the magnitude of the velocity vector (total velocity); the radial, tangential and axial components of the velocity vector at the exit of the rotor is provided in function of the span-wise distance.

Baseline Tips: Near the tip, where $r / R_{T i p} \geq 0.9$, the two base profiles show very similar trends in Figure 8. The total velocity is significantly reduced in the core of the passage where $r / R_{\text {Tip }} \leq 0.9$ when tip clearance is high at 0.135 " level. The $\mathrm{t}=0.100$ " and $\mathrm{t}=0.135$ " clearance base profiles show significant momentum deficit occurring in the core of the tip vortices from the two baseline tips (without any tip platforms). The tip vortices from the baseline cases greatly influence the core flow and reduce the mean kinetic energy when they are mixed with the wake fluid. The wake fluid and the tip leakage vortex is also modified and mixed by the serrated trailing edge geometry as shown in figure 2 . This observation indicates that a significant momentum deficit in the core of the passage vortex exists because of the tip vortex and this momentum deficit becomes higher when the clearance increases. The 
direct impact of the baseline tip vortex is visible in the measured total velocity in the core of the passage, even under strong mixing conditions induced by the wake fluid originating from the serrated trailing edges.

The Radial Component: The radial components for all cases as shown in figures 8 12 are all very small magnitudes around $\pm 1 \mathrm{~m} / \mathrm{s}$. There is no significant influence of the magnitude of baseline clearance or the type of the tip treatment on the magnitude of the radial component at all spanwise locations.

Influence of Tip Platform Extensions on the Tangential Component: When the axial velocity component in figure 9 is compared to the total velocity, a striking observation in the tip region is apparent. The total velocity for the baseline tips are much higher than the axial component where $r / R_{T i p} \geq 1.05$. However, the total velocity distribution for baseline tips is about the same as the axial velocity component for five treated tips shown in figure 8. Since the radial components are extremely small for all tip shapes, one can infer that the significant difference between the baseline tips and the treated tips is due to a strong change in the tangential component of the velocity vector. The distribution of the tangential component shows a significant difference in the whole measurement region where $r / R_{T i p} \geq 0.6$.

One can conclude that there is a strong swirl component near the tip region when there is no tip treatment. The five treated tips defined in figure 7 provide significant reduction in the amount of swirl in rotor exit flow. The reduction in the amount of swirl near the tip region is about $4-5 \mathrm{~m} / \mathrm{s}$ with the tip platform extensions shown in figure 7 . The magnitude of the swirl component that is inherent to baseline tips is about one third of the total velocity existing in the core of passage. 
Tip Platform Extensions with Highest Axial Velocity at Rotor Exit: Figure 9 shows that the five new tip platform extension devices can be highly instrumental in reducing the amount of swirl coming out of the rotor. This feature is certainly a benefit in terms of the energy efficiency of the axial flow fan when the fan is operated on its high volumetric flow rate (low pressure rise) point. The tip platform extensions help to reduce the tip leakage mass flow rate and its momentum deficit via local viscous flow modifications near the tip region. The Profile 1 and Profile 2 provide the two profiles with the highest total velocity in the core of the passage exit flow.

The Optimal Tip Platform Design: The experimental results suggest that "maximum width" of the bump is an important parameter in designing the tip de-sensitization geometry. The Profile 2 and 5 cover almost the same chordwise locations. The only difference between the two is the "maximum width" of the bump. Figures 8, 9 and10 clearly shows that the recovery of the axial velocity component and elimination of the tangential component is more effective with Profile 2 than with Profile 5. Figures 9,10 and 11 show that the features observed in figure 9 for location 3 repeat for other angular positions of the rotor (location 4 and 5). Although there are slight flow field differences at different rotor positions, the general nature of the discussion does not change.

Fan Exit Flow at the Highest Pressure Rise: An interesting tip treatment experiment could be performed by operating the fan at its highest pressure point by reducing the volumetric flow rate using a perforated steel plate at the mock-up inlet section as shown in figure 1. The experiments with the specific perforated plate with an open area ratio of $19.6 \%$ indicated that measurable gains in axial velocity component at the core of the exit flow still exist 
with Profile 2. Figure 12 shows that tangential and radial velocity components from Profile 2 are very similar to the baseline profile at the same effective clearance value of $0.100 "(1 \%)$. However the elimination of the tangential component observed in high volumetric flow rate experiments does not occur under high pressure rise conditions (140 Pa) as shown in Figure 12. There is a consistent $2 \mathrm{~m} / \mathrm{s}$ increase in axial (or total) velocity throughout the blade span when $r / R_{T i p} \geq 0.6$. Under high loading conditions the swirl component is minimal even with a baseline tip. The tip platform extensions for this case also perform their function by minimizing the tip leakage flow mass flow rate. The minimization of the tip leakage mass flow rate eventually provides the gain in axial (or total) velocity component. On the average, the gain in total velocity magnitude at the rotor exit is about $17 \%$ throughout the blade span. This type of gain in mean kinetic energy of fan exit flow is expected to contribute to the energy efficiency of the fan.

Rotor Exit Total Velocity Distributions: Figure 13 and 14 show total velocity contours drawn on axial and radial plane at the fan rotor exit for Baseline tip and Profile 2 respectively. Both contours are obtained from measurements performed at 0.1 " tip clearance. Rotor tip $\left(r / R_{\text {Tip }}=1.0\right)$ is marked with the dashed red line. An enhancement of total velocity near the tip region is observed, when tip region total velocities are compared. It is also obvious that using Profile 2 enhances the area coverage of high momentum jet at the exit. That means rotor blade with tip platform Profile 2 is increasing kinetic energy of the fluid flow more efficiently. It also reduces momentum deficit near the tip due to tip leakage flow. 


\section{Conclusions}

Novel tip platform extensions for energy efficiency gains and aeroacoustic improvements were designed for an axial flow fan where wake mixing is already enhanced with a serrated trailing edge design.

Five different tip platform extensions were introduced especially on the pressure side of the fan blades.

It is possible to reduce the tip leakage mass flow rate using the novel tip platform extensions. Profile 2 showed the best tip treatment performance out of the five new tip platform extensions designed in this investigation.

Tip treatment experiments performed at high volumetric flow rate/low pressure rise clearly showed the minimization of the tip vortex mass flow rate by significantly reducing the tangential components near the tip.

The tip platform extensions on the pressure side have proven to be effective swirl reducing devices at the exit of the fan. The magnitude of this reduction is about one third of the rotor exit total velocity in the core of the passage exit.

The reduction of tip leakage mass flow rate produced enhanced total velocity values between the mid-span and the tip at the passage exit.

Tip platform experiments performed at the highest pressure point by reducing the volumetric flow rate using a perforated steel plate at the mock-up inlet section also showed significant tip leakage control for the fan. However the elimination of the tangential component observed in high volumetric flow rate experiments does not occur under high pressure rise conditions. 
Under high loading conditions the swirl component is minimal even with a baseline tip. With the tip platform extension "Profile 2", there is a consistent $2 \mathrm{~m} / \mathrm{s}$ increase in axial (or total) velocity throughout the blade span when $r / R_{T i p} \geq 0.6$.

Tip platform extensions perform their function by minimizing the tip leakage flow mass flow rate near the blade tip even under the high loading conditions. This effect eventually provides the gain in axial component.

On the average, the gain in total velocity magnitude at the rotor exit is about $17 \%$ throughout the blade span. This type of gain in mean kinetic energy of fan exit flow is expected to contribute to the energy efficiency of the fan.

\section{Acknowledgments}

The authors acknowledge the major support from VLRCOE (Vertical Lift Rotorcraft Center of Excellence) of the Department of Aerospace Engineering at Penn State University. This research is partially funded by the Government under Agreement No. W911W6-06-20008. The U.S. Government is authorized to reproduce and distribute reprints notwithstanding any copyright notation thereon. The views and conclusions contained in this document are those of the authors and should not be interpreted as representing the official policies,

either expressed or implied, of the U.S. Government. The authors also acknowledge the technical support of Mr.H.Houtz during the experiments. 


\section{References}

[1] Lee, G. H., Baek, J. H., and Myung, H. J., 2003, "Structure of tip leakage in a forwardswept axial-flow fan," Flow, Turbulence and Combustion, 70, pp. 241-265.

[2] Jang, C. M., Furukawa, M., and Inoue, M., 2001, "Analysis of vertical flow field in a propeller fan by ldv measurements and les - parts i, ii," Journal of Fluids Engineering, 123, pp. 748-761.

[3] Storer, J. A. and Cumpsty, N. A., 1991, "Tip leakage flow in axial compressors," Journal of Turbomachinery, 113.

[4] Lakshminarayana, B., Zaccaria, M., and Marathe, B., 1995, "The structure of tip clearance flow in axal flow compressors," Journal of Turbomachinery, 117, pp. 336-347.

[5] Inoue, M., Kuroumaru, M., and Furukawa, M., 1986, "Behavior of tip leakage flow behind an axial compressor rotor," Journal of Gas Turbine and Power, 108, pp. 7-14.

[6] Furukawa, M., Inoue, M., Kuroumaru, M., Saik, i. K., and Yamada, K., 1999, "The role of tip leakage vortex breakdown in compressor rotor aerodynamics," Journal of Turbomachinery, 121, pp. 469-480.

[7] Fujita, H. and Takata, H., 1984, "A study on configurations of casing treatment for axial flow compressors," Bulletin of the JSME, 27, pp. 1675-1681.

[8] Moore, R. D., Kovich, G., and Blade, R. J., 1971, "Effect of casing treatment on overall and blade-element performance of a compressor rotor," Tech. Rep. TN-D6538, NASA. 
[9] Reynolds, B., Lakshminarayana, B., and Ravindranath, A., 1979, "Characteristics of near wake of a fan rotor blade," AIAA Journal, 17, pp. 959-967.

[10] Ravindranath, A. and Lakshminarayana, B., 1980, "Mean velocity and decay characteristics of near and far-wake of a compressor rotor blade of moderate loading," Journal of Engineering for Power, 102, pp. 535-547.

[11] Myung, H. J. and Baek, J. H., 1999, "Mean velocity characteristics behind a forwardswept axial-flow fan," JSME Int. J., 42, pp. 476-488.

[12] Adrian, R., 1991, "Particle imaging techniques for experimental fluid mechanics," Ann. Rev. Fluid Mech., 23, pp. 261-304.

[13] Kahveci, H. S. and Camcı, C., 2006, "Flow around helicopter blade tip sections using 2D particle image velocimeter-part I," 11th Int. Symp. on Transport Phenomena and Dynamics of Rotating Machinery (ISROMAC-11), (136).

[14] Kahveci, H. S. and Camc1, C., 2006, "Flow around helicopter blade tip sections using a (3D) stereoscopic particle image velocimeter-part II," 11th Int. Symp. on Transport Phenomena and Dynamics of Rotating Machinery (ISROMAC-11), (137).

[15] Kahveci, H. S., 2004, Implementation of a Stereoscopic PIV in Rotating Machinery Including Helicopter Rotor Flows, MS Thesis, The Pennsylvania State University, University Park, PA.

[16] Yoon, J. H. and Lee, S. J., 2004, "Exit flow field and performance of axial flow fans," Experimental Thermal and Fluid Science, 28, pp. 791-802. 
[17] Yen, S. C. and Lin, F. K. T., 2006, "Exit flow field and performance of axial flow fans," Journal of Fluids Engineering, 128, pp. 332-340.

[18] Wernet, M. P., Van Zante, D., Strazisar, T. J., John, W. T., and Prahst, P. S., 2005, "Characterization of tip clearance flow in an axial compressor using 3-d digital piv," Experiments in Fluids, 39, pp. 743-753.

[19] Corsini, A., Perugini, B., Rispoli, F., Kinghorn, I., and Sheard, A. G., 2006, "Investigation on improved blade tip concept," ASME Paper, (GT2006-90592).

[20] Corsini, A., Rispoli, F., and Sheard, A. G., 2006, "Development of improved blade tip end-plate concepts for low-noise operation in industrial fans," Proceedings of Conference on Modeling Fluid Flows CMMF06.

[21] Corsini, A., Perugini, B., Rispoli, F., Kinghorn, I., and Sheard, A. G., 2007, "Experimental and numerical investigations on passive devices for tip-clearance induced noise reduction in axial flow fans," Proceedings of the 7th European Conference on Turbomachinery.

[22] Corsini, A., Perugini, B., Rispoli, F., Sheard, A. G., and Kinghorn, I., 2007, "Aerodynamic workings of blade tip end-plates designed for low-noise operation in axial flow fans," ASME Paper, (GT2007-27465).

[23] Ott, R. L. and Longnecker, M. T., 2000, An Introduction to Statistical Methods and Data Analysis, Duxbury Press, California. 


\section{List of Figures}

1. Figure 1: Test rig and stereoscopic SPIV setup

2. Figure 2: Axial flow fan as seen from the exit plane and the SPIV system orientation

3. Figure 3: Geometric and blade section parameters of axial flow fan

4. Figure 4: Axial flow fan performance

5. Figure 5: SPIV measurement plane (horizontal) downstream of the rotor exit and the coordinate system

6. Figure 6: Influence of sample size in SPIV ensemble averaging

7. Figure 7: Novel tip platform extensions for tip leakage mitigation

8. Figure 8: Velocity profiles measured at location 3 for two different tip clearances

9. Figure 9: Velocity profiles measured at location $3\left(340 \mathrm{~m}^{3} / \mathrm{min}, 32 \mathrm{~Pa}\right)$

10. Figure 10: Velocity profiles measured at location $4\left(340 \mathrm{~m}^{3} / \mathrm{min}, 32 \mathrm{~Pa}\right)$

11. Figure 11: Velocity profiles measured at location $5\left(340 \mathrm{~m}^{3} / \mathrm{min}, 32 \mathrm{~Pa}\right)$

12. Figure 12: Velocity profiles measured at location 3 (high $\Delta \mathrm{P}$ with $19.6 \%$ perforated plate, $80 \mathrm{~m}^{3} / \mathrm{min}, 140 \mathrm{~Pa}$ )

13. Figure 13: Total velocity contour and streamlines at location 3 for baseline profile (340 $\left.m^{3} / \min , 32 \mathrm{~Pa}\right)$

14. Figure 14: Total velocity contour and streamlines at location 3 for $2^{\text {nd }}$ profile (340 $\left.m^{3} / \min , 32 \mathrm{~Pa}\right)$ 


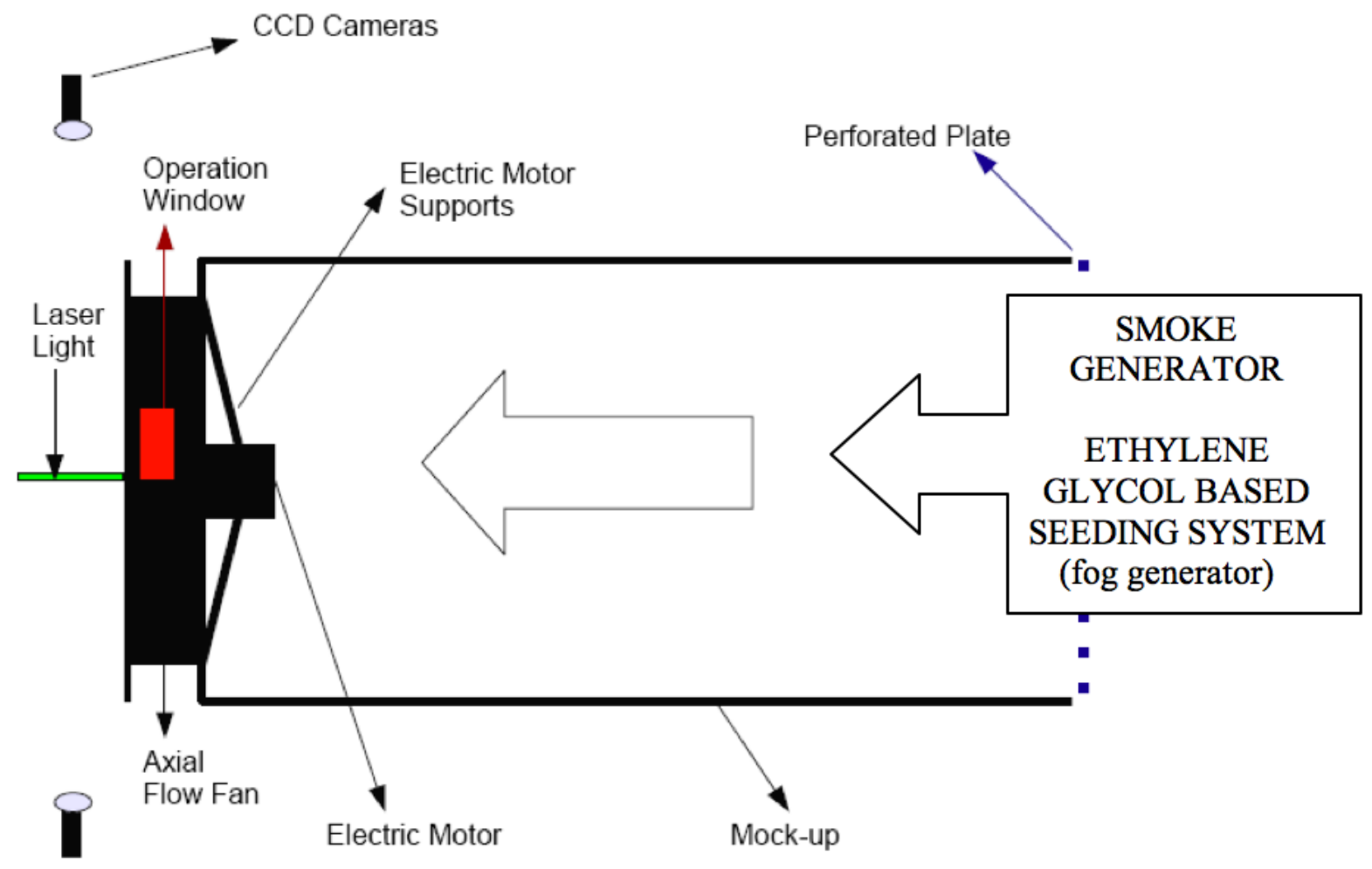

Figure 1: Test rig and stereoscopic SPIV setup 


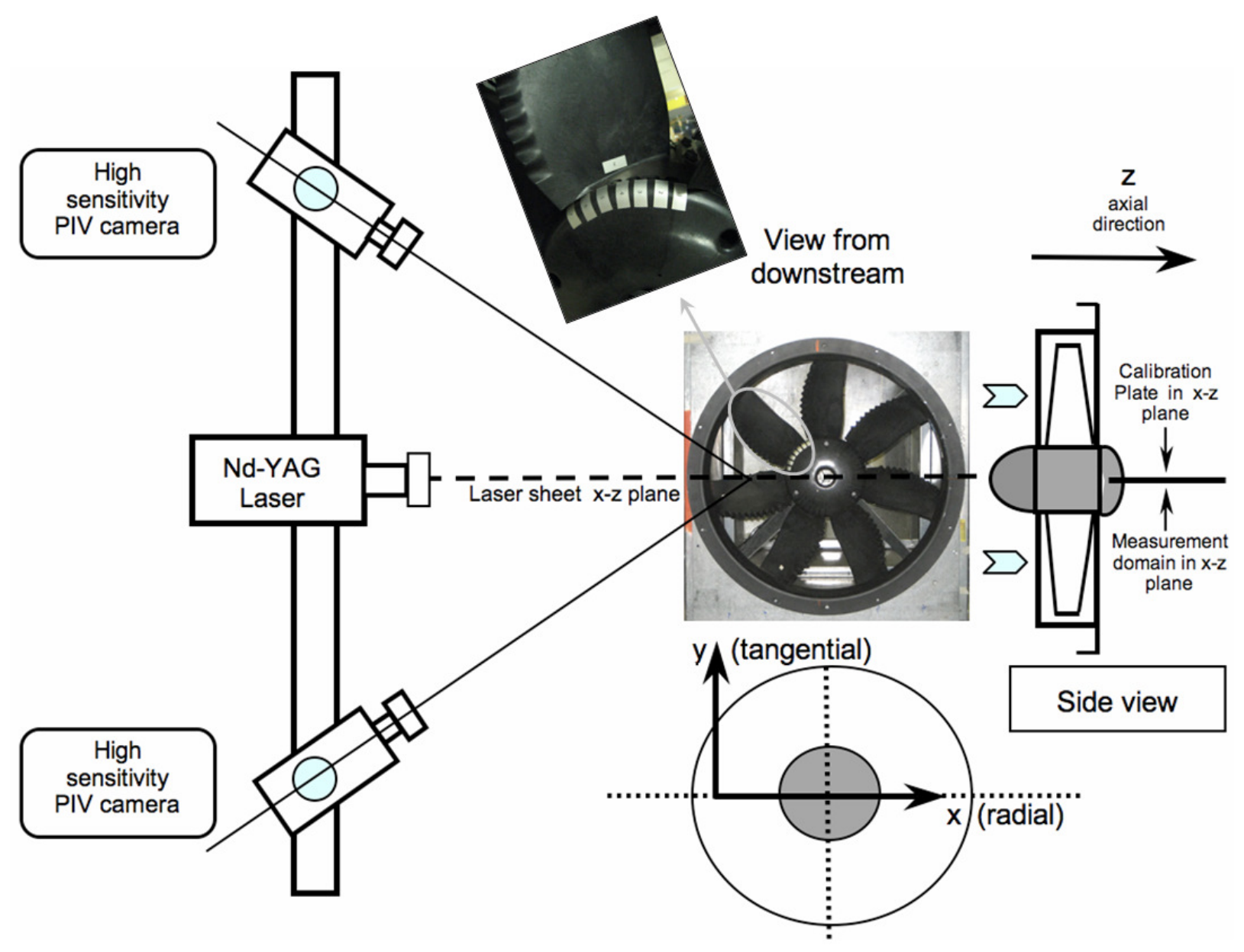

Figure 2: Axial flow fan as seen from the exit plane and the SPIV system orientation 


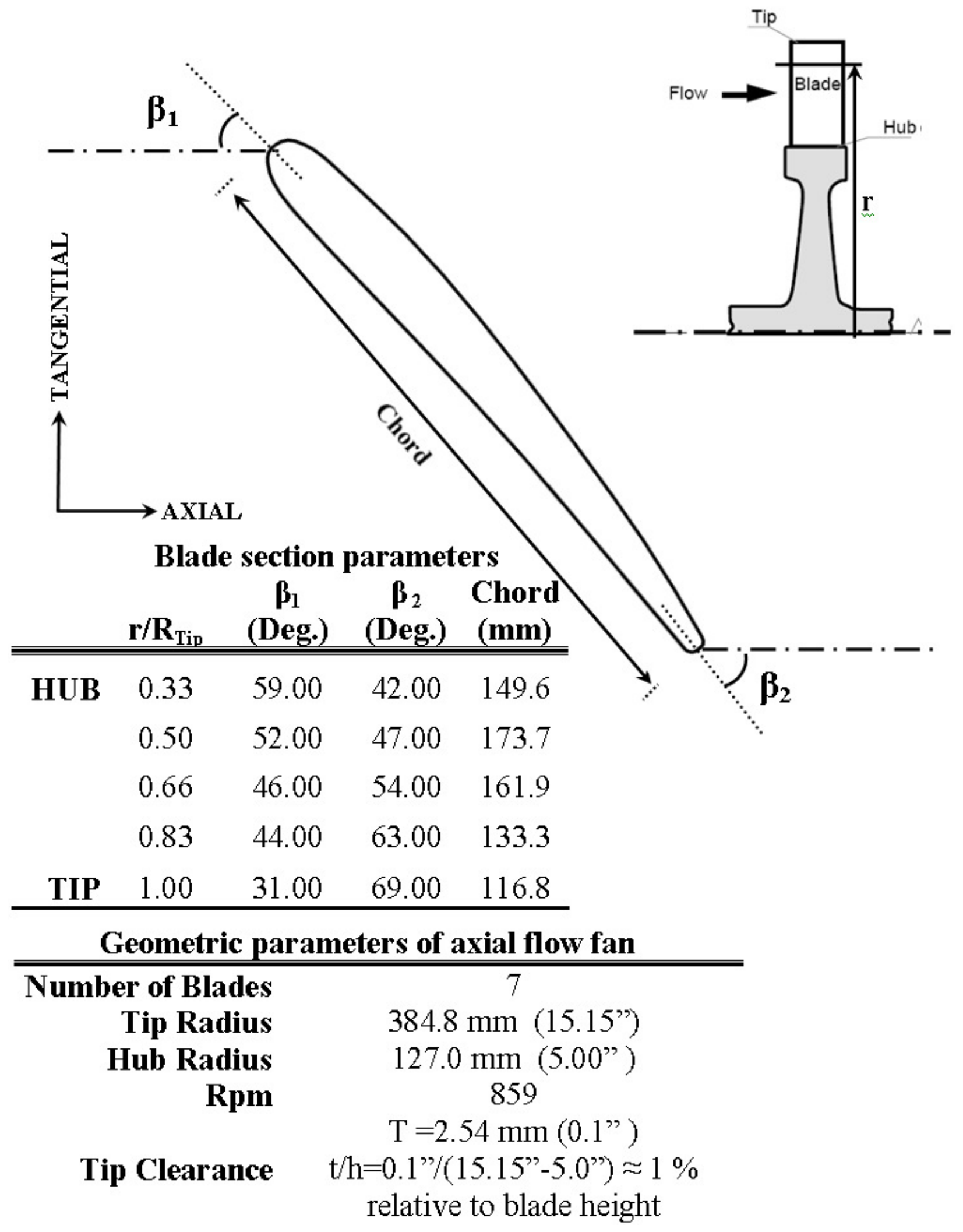

Figure 3: Geometric and blade section parameters of axial flow fan 


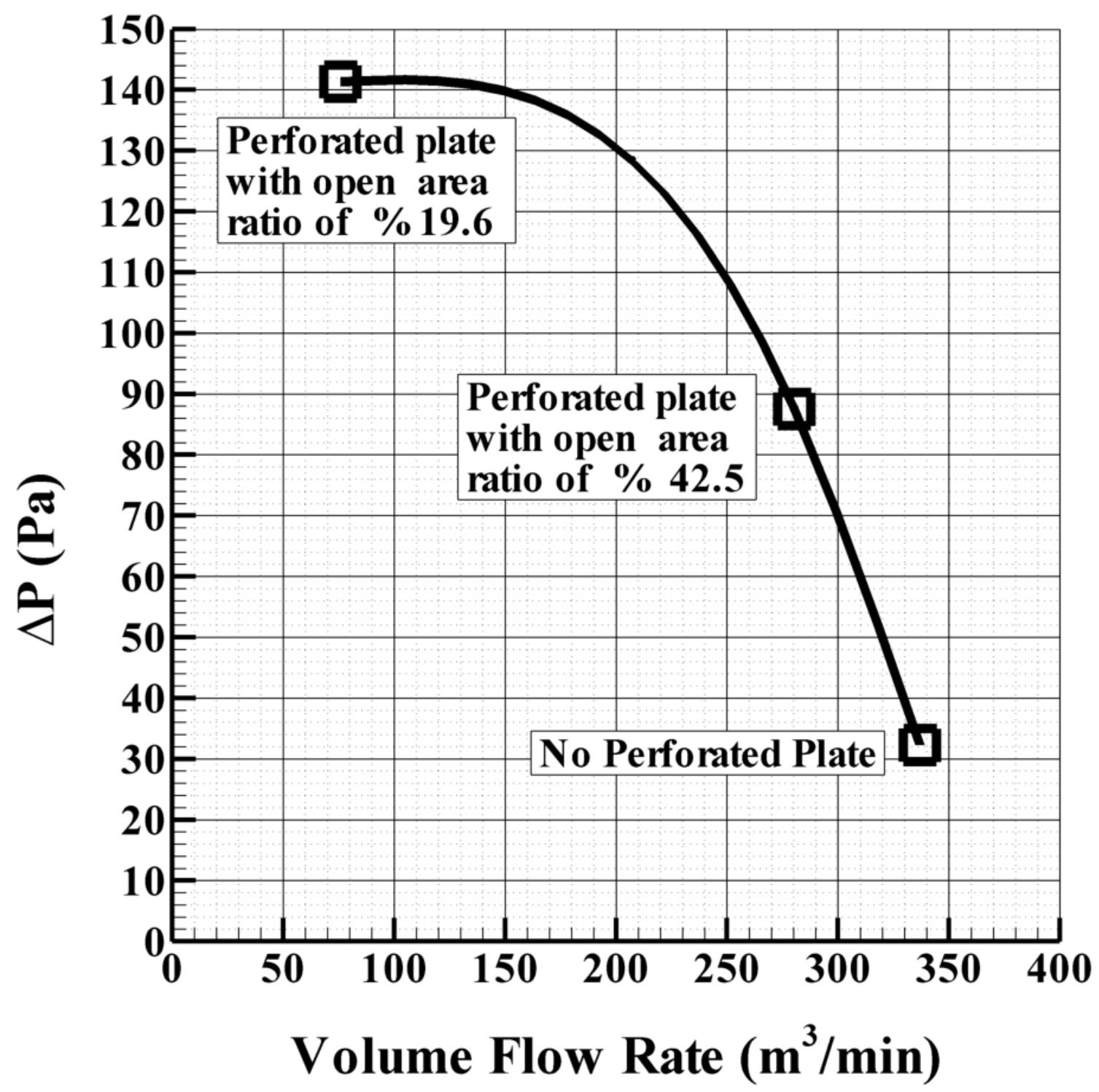

Figure 4: Axial flow fan performance 
ROTOR EXIT PLANE

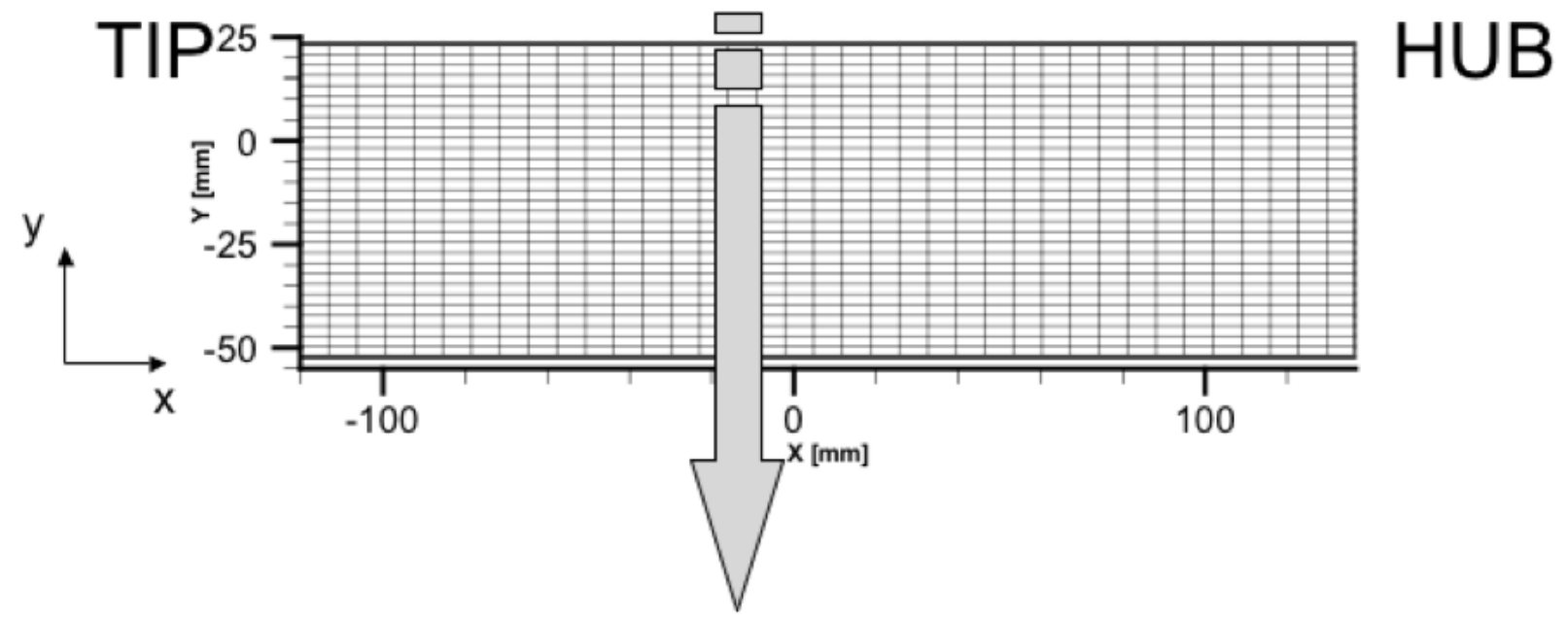

Figure 5: SPIV measurement plane (horizontal) downstream of the rotor exit and the coordinate system 


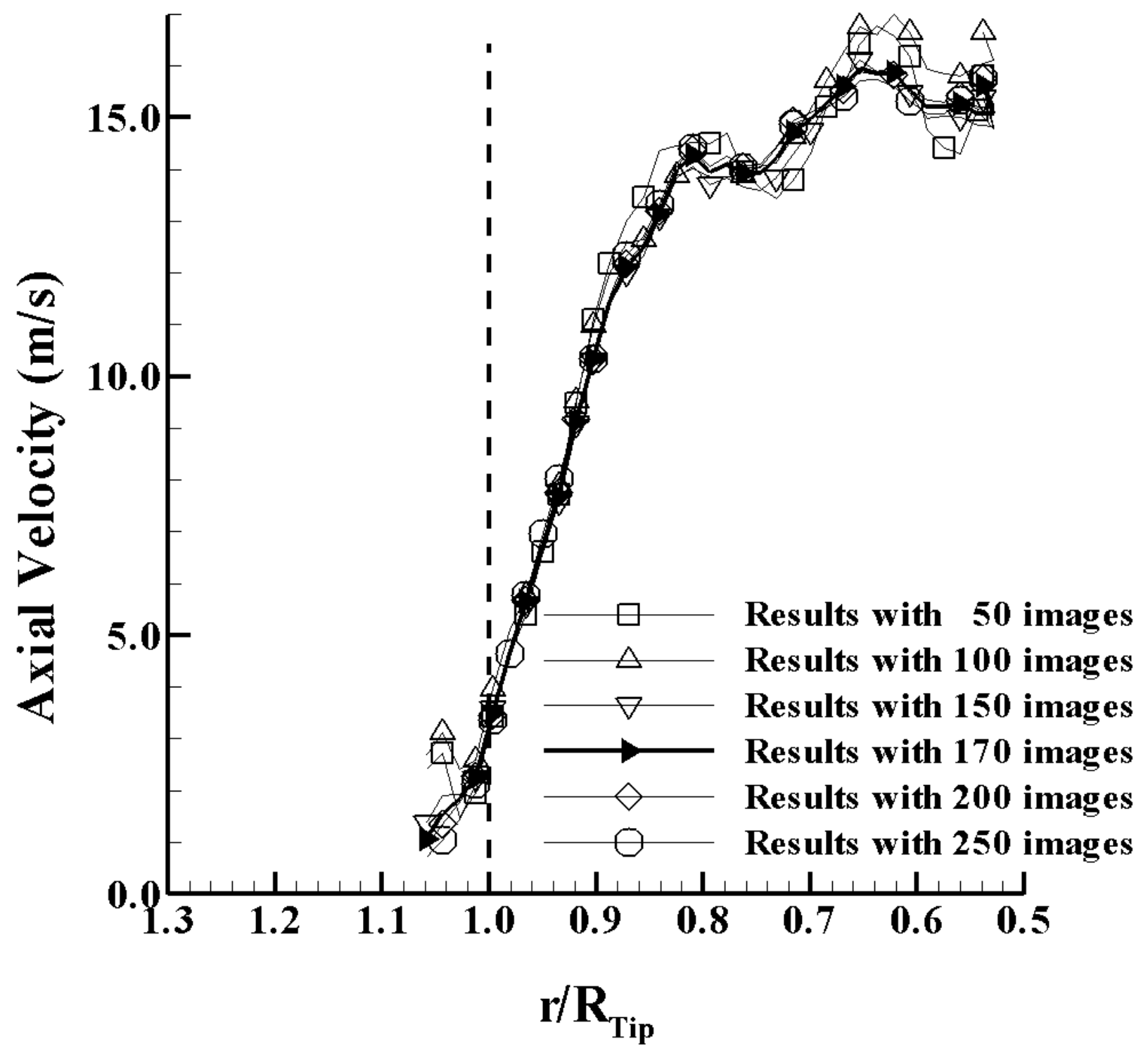

Figure 6: Influence of sample size in SPIV ensemble averaging 


\section{Baseline Profile}

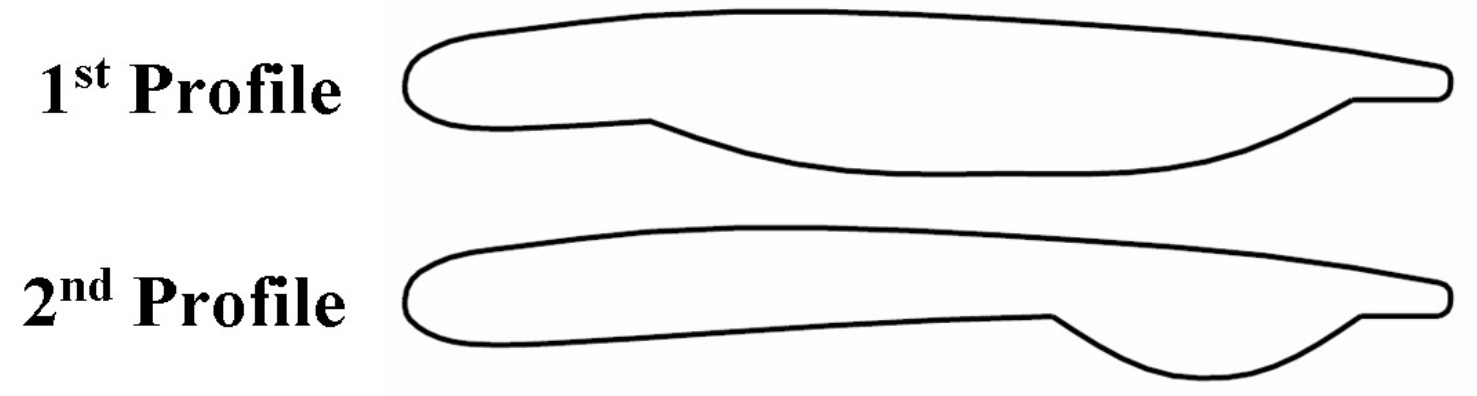

$3^{\text {rd }}$ Profile

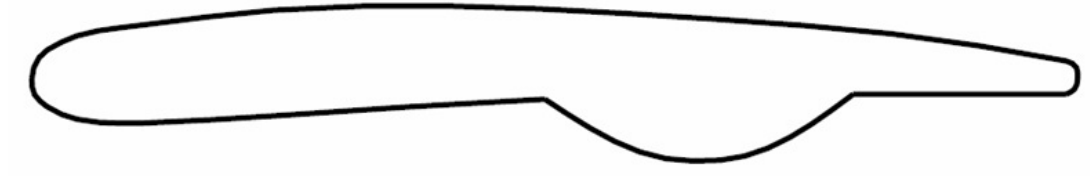

$4^{\text {th }}$ Profile

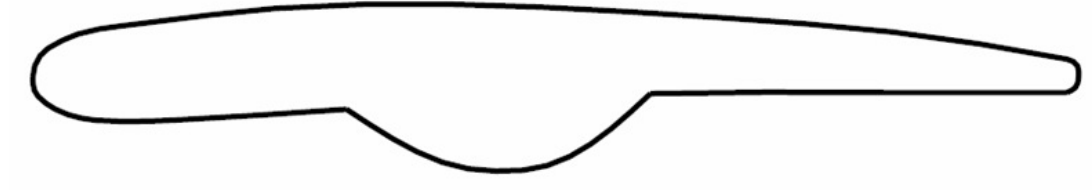

$5^{\text {th }}$ Profile

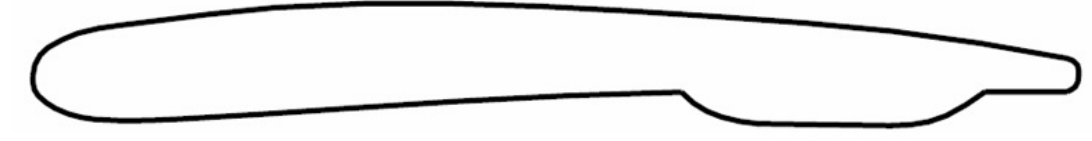

Figure 7: Novel tip platform extensions for tip leakage mitigation 

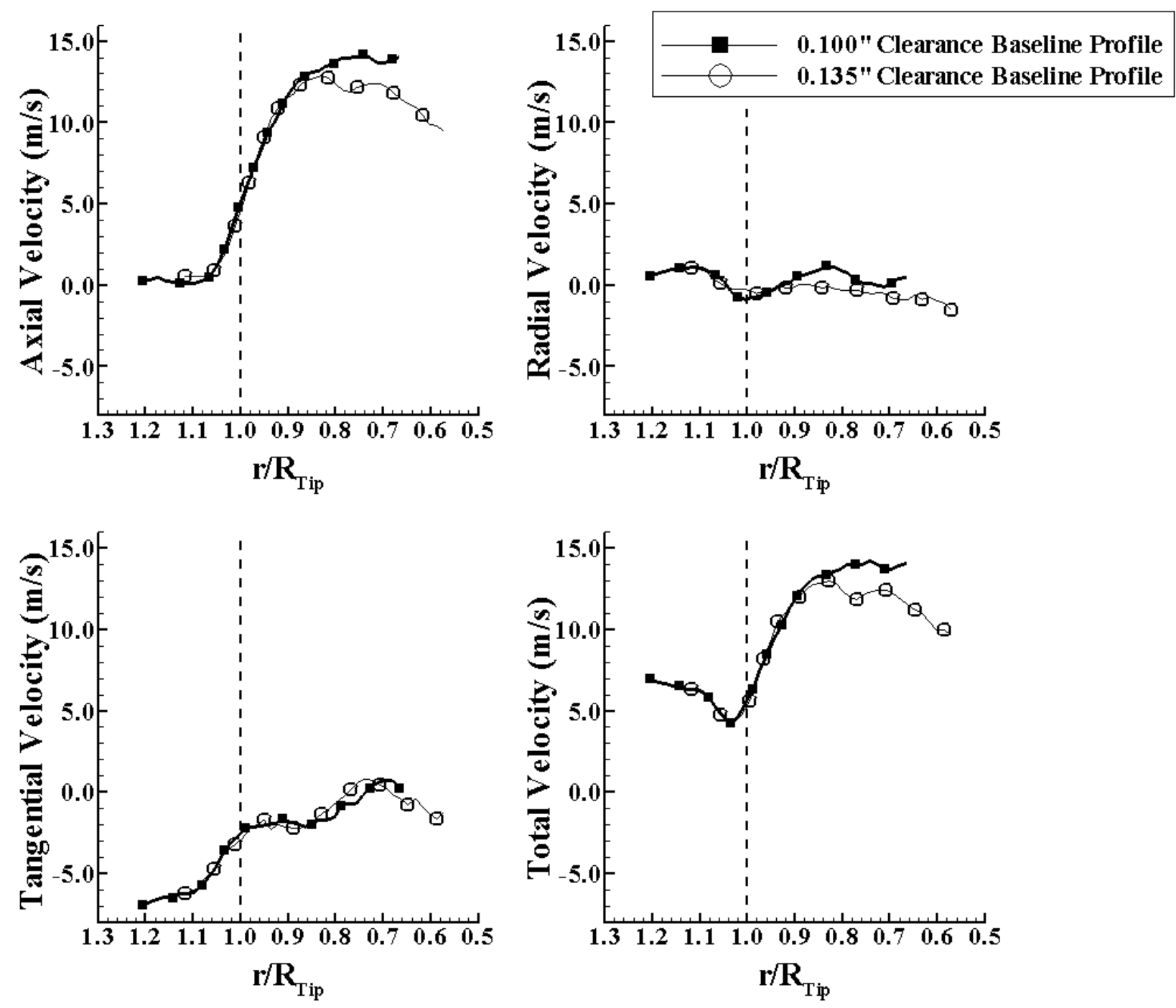

Figure 8: Velocity profiles measured at location 3 for two different tip clearances 

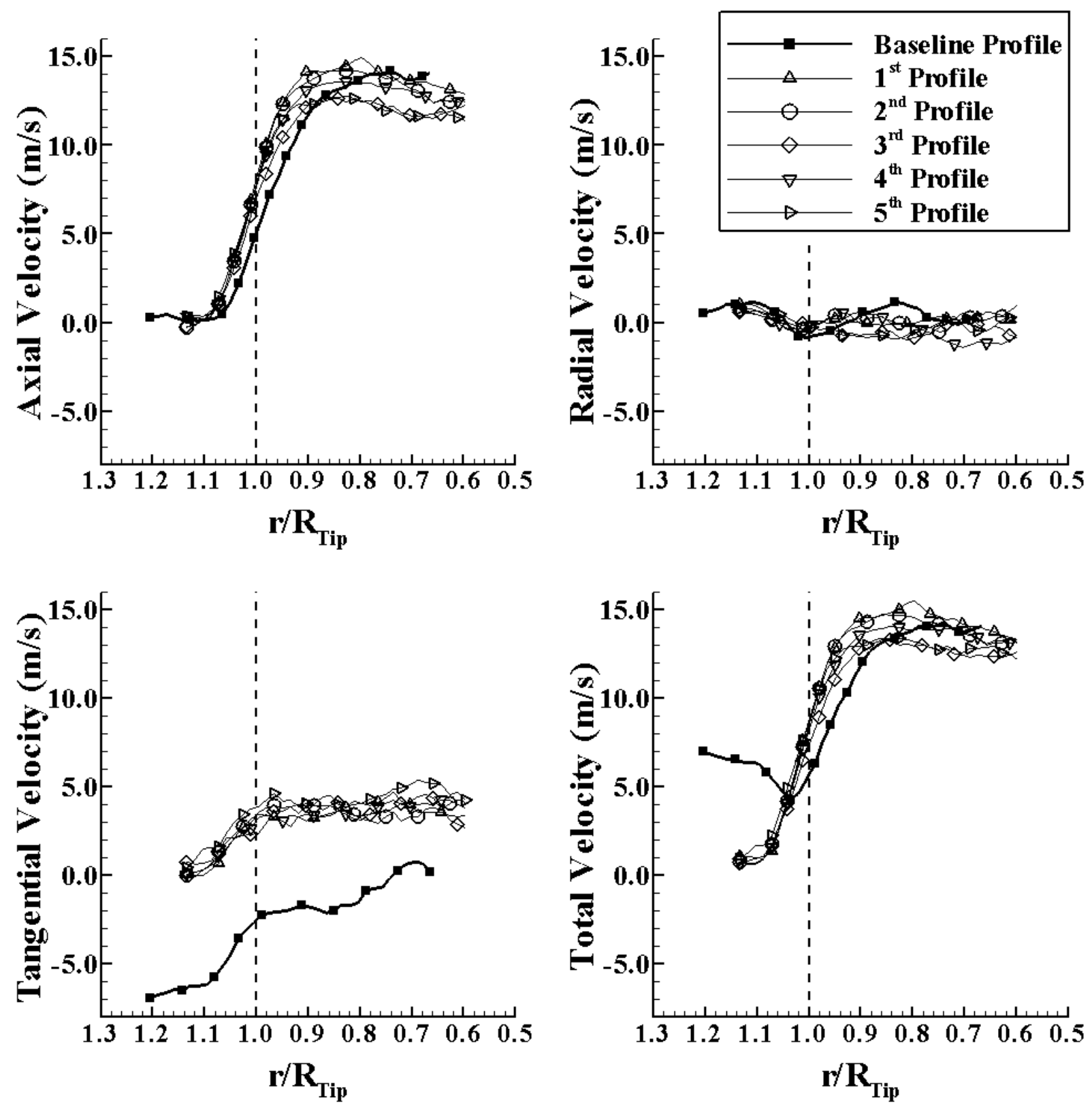

Figure 9: Velocity profiles measured at location $3\left(340 \mathrm{~m}^{3} / \mathrm{min}, 32 \mathrm{~Pa}\right)$ 

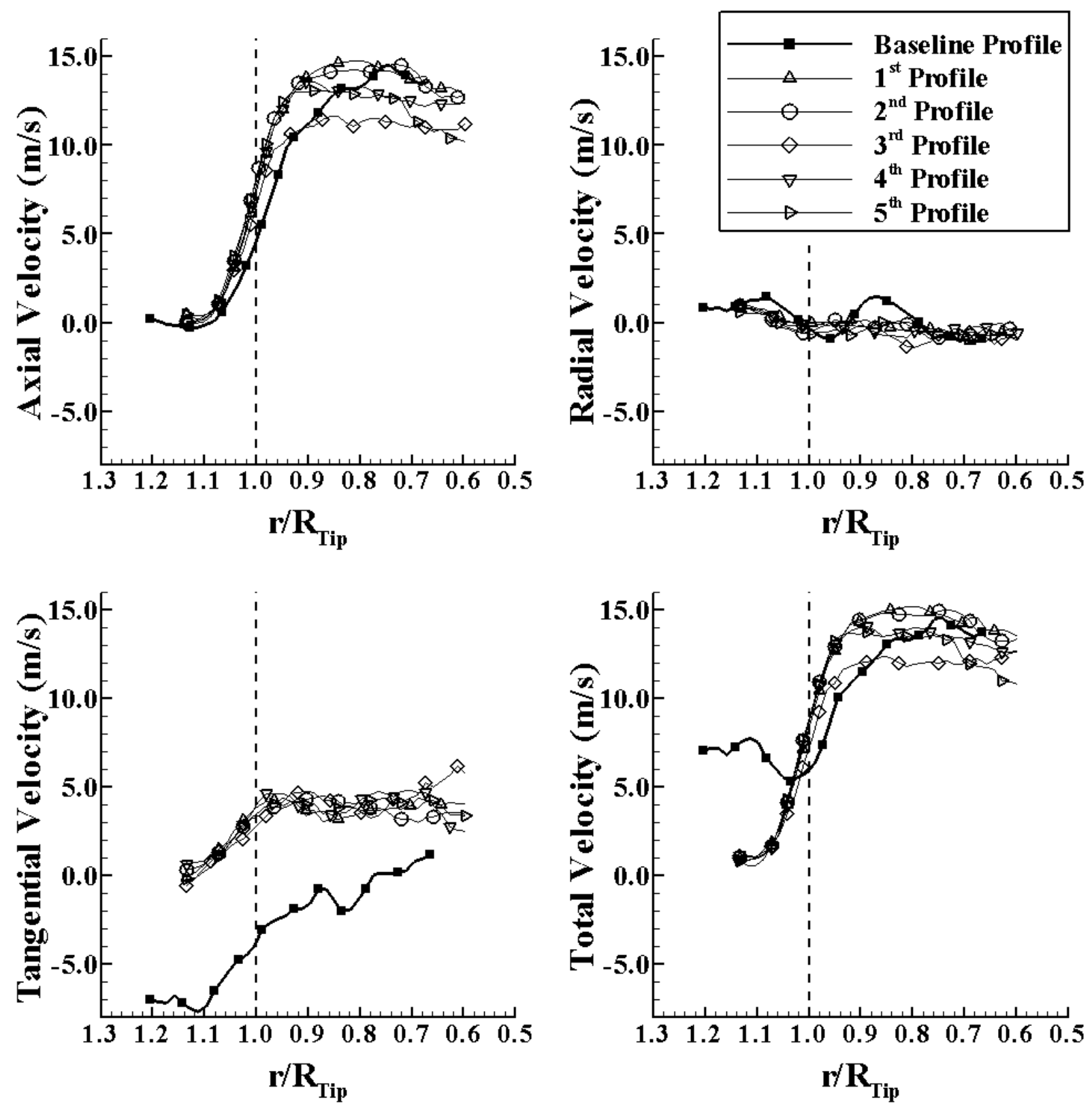

Figure 10: Velocity profiles measured at location $4\left(340 \mathrm{~m}^{3} / \mathrm{min}, 32 \mathrm{~Pa}\right)$ 

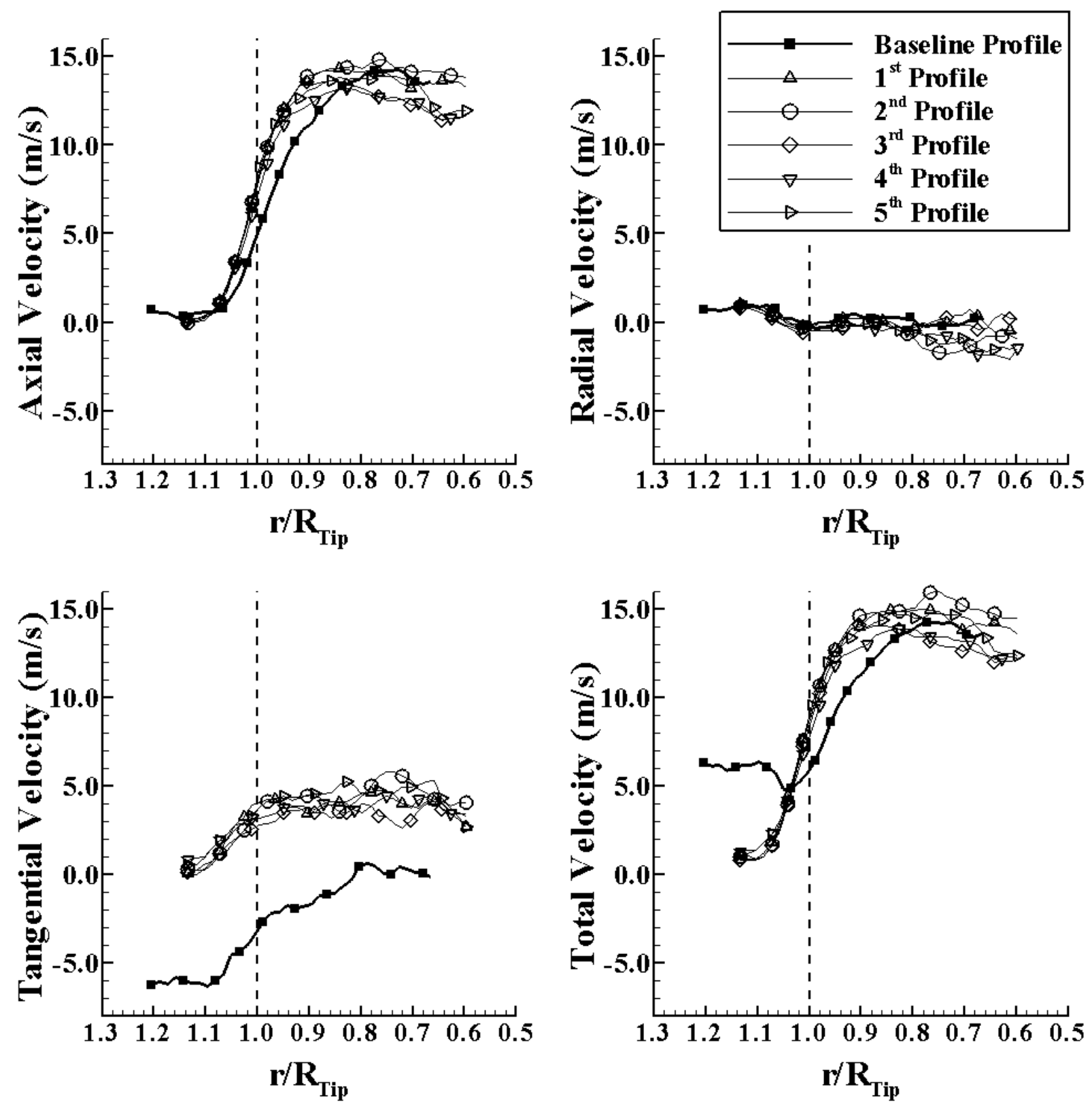

Figure 11: Velocity profiles measured at location $5\left(340 \mathrm{~m}^{3} / \mathrm{min}, 32 \mathrm{~Pa}\right)$ 

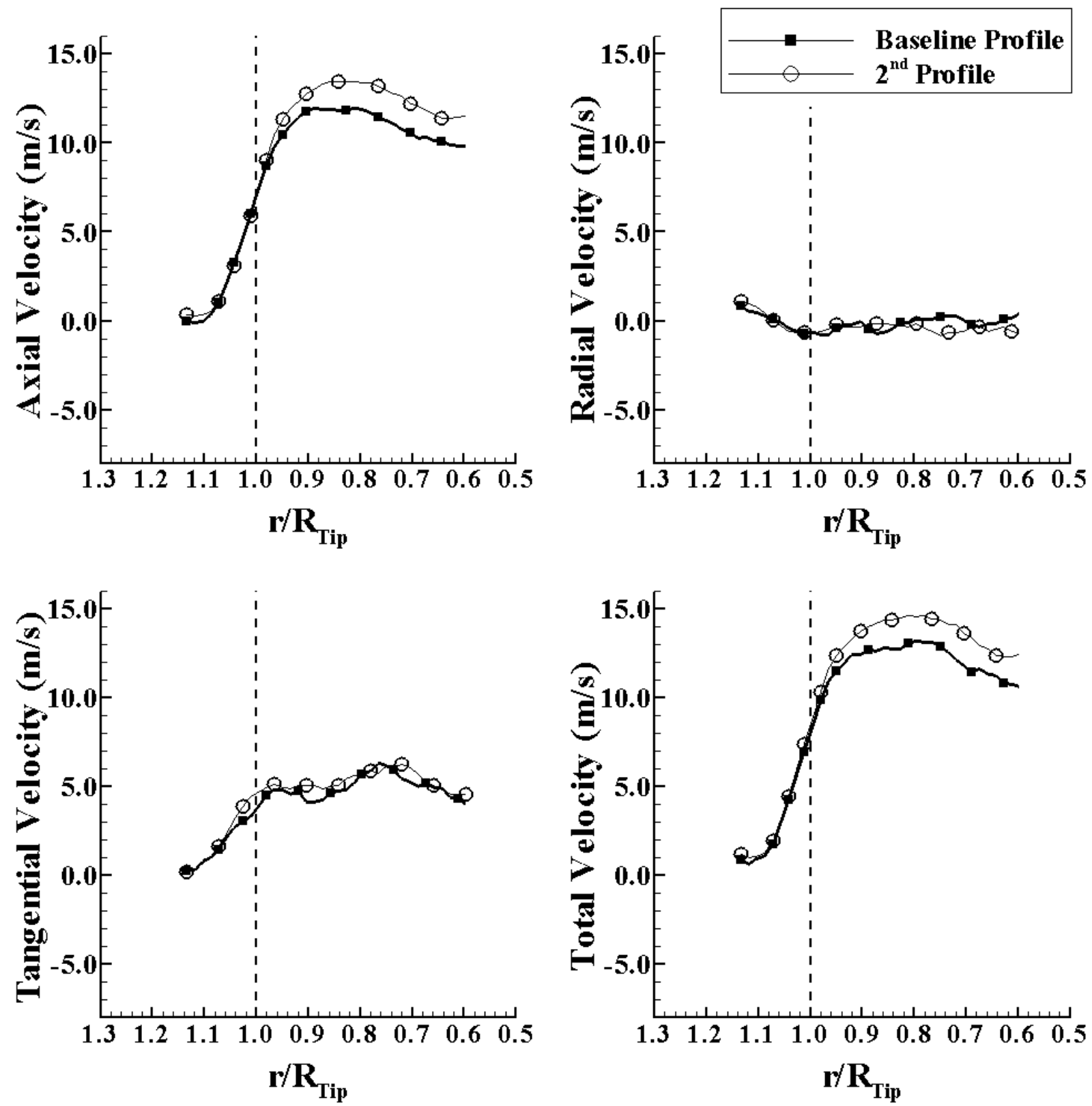

Figure 12: Velocity profiles measured at location 3 (high $\Delta \mathrm{P}$ with $19.6 \%$ perforated plate, $\left.80 \mathrm{~m}^{3} / \mathrm{min}, 140 \mathrm{~Pa}\right)$ 


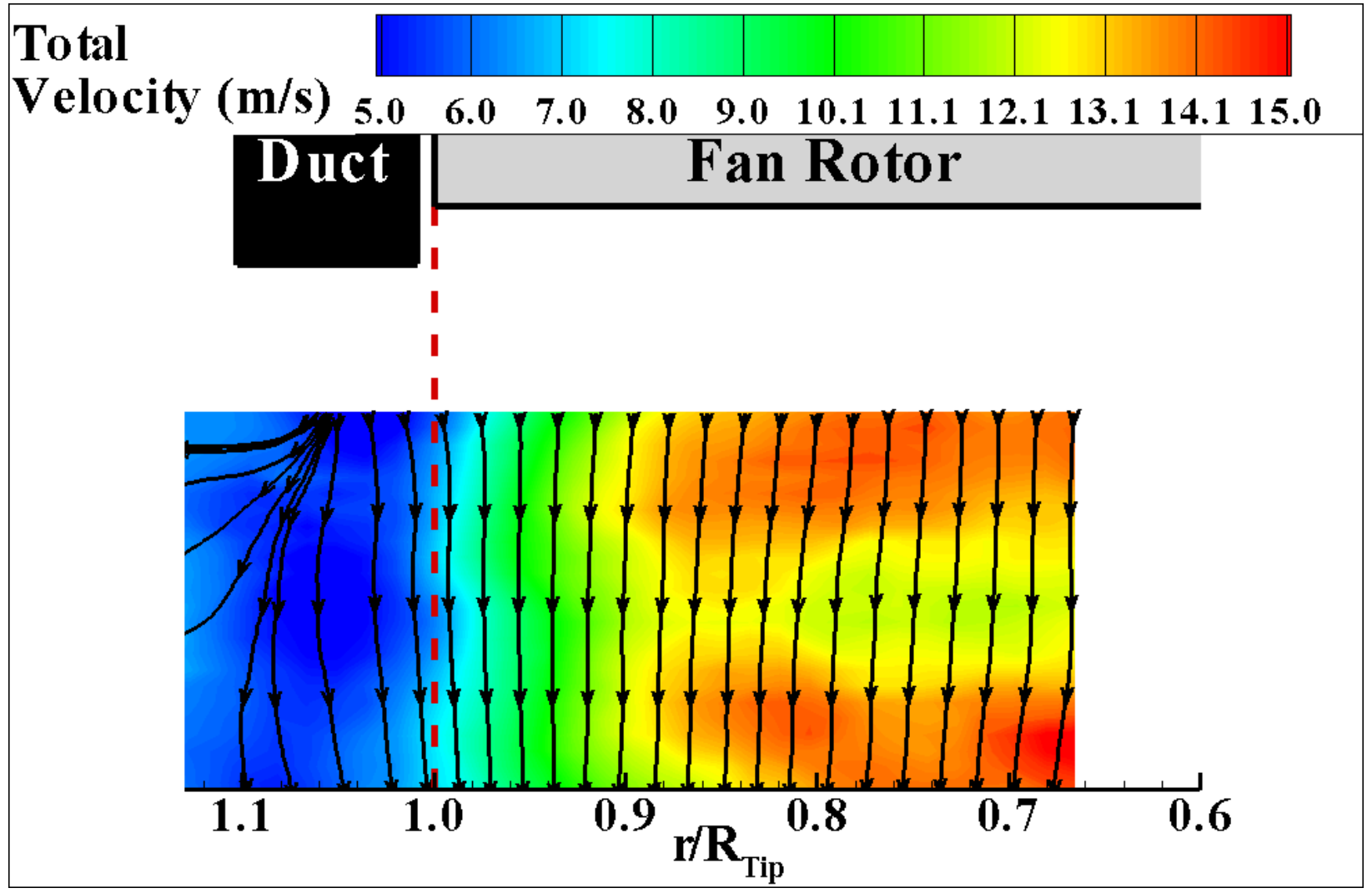

Figure 13: Total velocity contour and streamlines at location 3 for baseline profile (340 $\left.\mathrm{m}^{3} / \mathrm{min}, 32 \mathrm{~Pa}\right)$ 


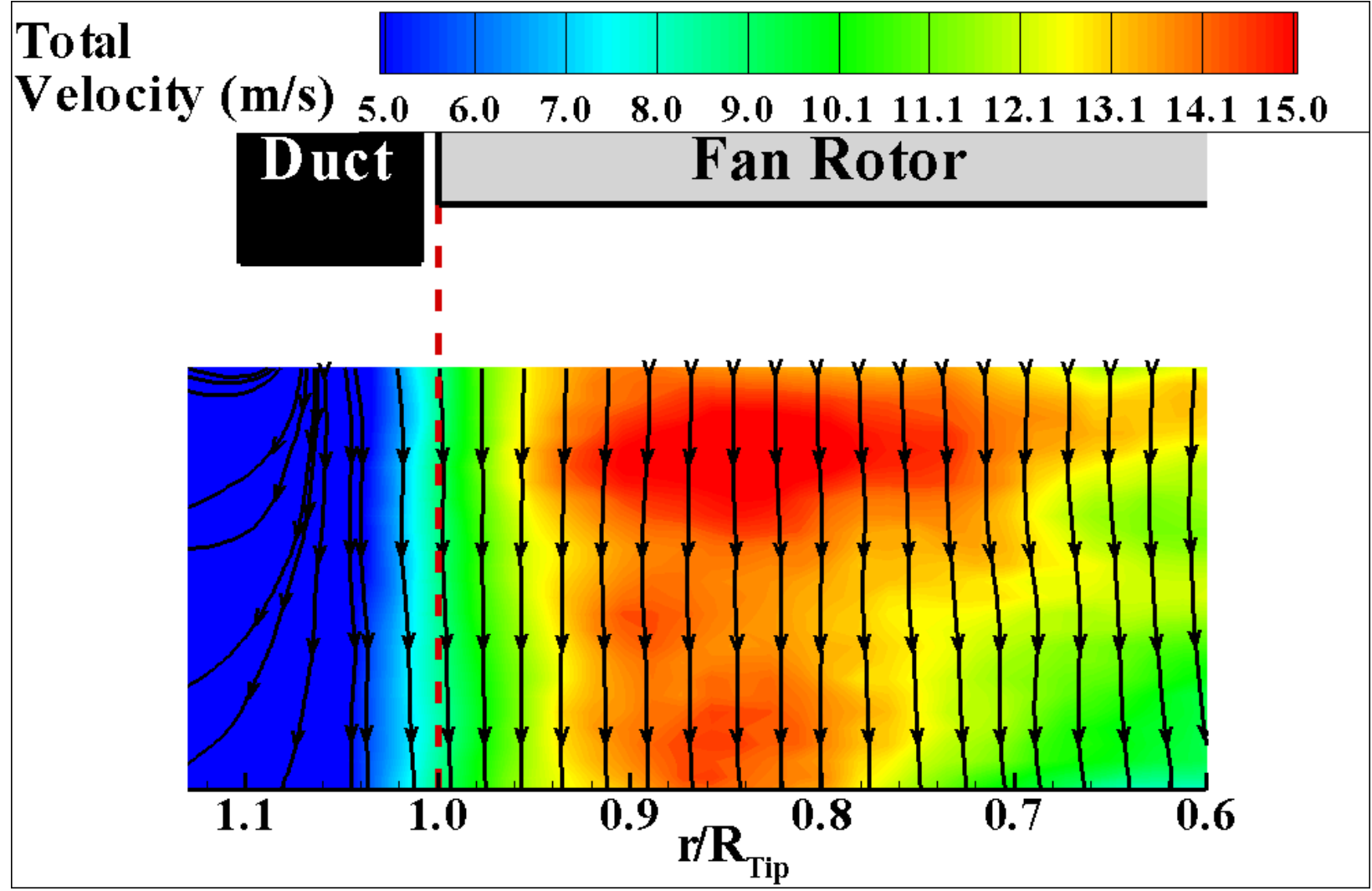

Figure 14: Total velocity contour and streamlines at location 3 for $2^{\text {nd }}$ profile $\left(340 \mathrm{~m}^{3} / \mathrm{min}\right.$, $32 \mathrm{~Pa})$ 